\title{
SOME MODIFIED EIGENVALUE PROBLEMS
}

G.H. Golub

In many applications, various eigenvalue problems arise which are slightly nonstandard. That is, the usual algorithms for computing eigensystems are not directy applicable. For instance, in various statistical data-fitting problems, it is desirable to find ${ }_{x \neq 0} \frac{x^{\prime} A x}{x^{\prime} B x}$ subject to the constraint $C^{\prime} x=0$. In this talk we shall present an algorithm for reducing this problem to the usual eigenvalue problem. In addition, we shall consider methods for solving the eigenvalue problem $A x=\lambda B x$ when $A$ and $B$ are singular and we shall consider the problem of determining the eigensystem of a matrix which has been modified by a matrix of rank one. 